\title{
Reduced Expression of RanBPM Is Associated with Poorer Survival from Lung Cancer and Increased Proliferation and Invasion of Lung Cancer Cells In Vitro
}

\author{
ZEHANG ZHAO ${ }^{1,2}$, SHAN CHENG ${ }^{1}$, CATHERINE ZABKIEWICZ $^{2}$, JINFENG CHEN $^{3}$, \\ LIJIANG ZHANG ${ }^{3}$, LIN YE ${ }^{2}$ and WEN G. JIANG ${ }^{1,2}$ \\ ${ }^{1}$ Cardiff University-Capital Medical University Joint Centre for Biomedical Research \& Cancer Institute, \\ Capital Medical University, Beijing, P.R. China; \\ ${ }^{2}$ Cardiff China Medical Research Collaborative, Cardiff University School of Medicine, Cardiff, U.K.; \\ ${ }^{3}$ Key Laboratory of Carcinogenesis and Translational Research, Ministry of Education, Department of Thoracic Surgery II, \\ Beijing Cancer Hospital \& Institute, Peking University School of Oncology, Beijing, P.R. China
}

\begin{abstract}
Background/Aim: Ran binding protein microtubule-organizing centre (RanBPM), also known as RanBP9, is a scaffold protein conserved through evolution. We investigated the role of RanBPM in human lung cancer. Materials and Methods: Transcripts of RanBPM were determined in 56 human lung cancers along with paired normal lung tissues using real-time PCR. Association with prognosis was analyzed by online Kaplan-Meier survival analysis. In vitro lung cancer cell functional assays examined the impact of RanBPM-knockdown on cellular growth and invasion. Results: Higher expression of RanBPM was observed in tumor when compared to paired normal lung tissues. Increased RanBPM expression was seen in patients with longer overall and disease-free survival. Knockdown of RanBPM in lung cancer cell lines resulted in increased growth and invasion in vitro. Conclusion: Increased expression of RanBPM associates with postponed disease progression and better prognosis. RanBPM plays an inhibitory role in regulating proliferation and invasion of lung cancer cells.
\end{abstract}

Lung cancer is the most common cancer and the leading cause of cancer-related death in China and worldwide $(1,2)$. Lung cancers develop through a multistep process involving development of multiple genetic and epigenetic alterations,

Correspondence to: Prof. Wen G. Jiang, Cardiff China Medical Research Collaborative, Institute of Cancer and Genetics, Cardiff University School of Medicine, Heath Park, Cardiff, CF14 4XN, U.K. Tel: +44 2920687065, e-mail: JiangW@cf.ac.uk

Key Words: RanBPM, lung cancer, proliferation, adhesion, migration, invasion. particularly activation of growth-promoting pathways and inhibition of tumor-suppressor pathways (3-6). It is critical to have a better understanding of the genetic and molecular machinery involved in the tumorigenesis and disease progression of lung cancer for future targeted therapy (7-9). Unfortunately, little progress has been made in the treatment of advanced or metastatic lung cancer due to remaining gaps in the knowledge of the mechanisms of the disease (10-12). Therefore, there has been great interest in the identification of novel molecular targets or biomarkers to facilitate early diagnosis and the development of novel biological therapies.

Ran binding protein microtubule-organizing centre (RanBPM), also known as RanBP9 functions as a developmentally-conserved scaffold protein. It is a universally expressed nucleocytoplasmic protein. The RanBPM gene is located on human chromosome $6 \mathrm{p} 23$ encoding a $77.8 \mathrm{kDa}$ protein $(13,14)$. The RanBPM protein contains several conserved domains that provide potential protein-protein interaction sites, such as a spore lysis $\mathrm{A}$ and ryanoine receptor (SPRY) domain that is proposed to function as a proteininteraction module $(15,16)$, a $\mathrm{N}$-terminus proline rich domain (PRD), a lissencephaly type-I-like homology (LisH) motif, a CT11-RanBPM (CRA) motif and a carboxy-terminal to LisH (CTLH) motif at the C terminus of RanBPM (17). RanBPM regulates numerous cellular processes by interacting with different proteins, such as gene expression, signal transduction, cell adhesion, invasion and migration. To date, its biological functions remain largely unknown (18-22).

In human and murine cell lines, RanBPM is ubiquitously expressed and has been detected at the nucleus, cytoplasm, plasma membrane, and cell junctions (23-26). In an exploration of its involvement in malignancies, RanBPM has been shown to be a negative regulator of BLT2 (leukotriene B4 receptor 2) which inhibits interleukin 8 (IL-8) production 
and reactive oxygen species (ROS) generation leading to an increase of invasiveness and other aggressive traits of breast cancer cells (21). Similarly, its expression is also associated with distant metastasis in gastric cancers. Our recent study has shown that gastric cancers with distant metastases have lower levels of RanBPM expression compared to nonmetastatic tumors (20). Finally, there is a recent study whereby RanBPM acts as a mediator of the cellular DNA damage response (DDR), and accumulates in the nucleus upon interventional radiation (IR), depending on ATM kinase activity in lung cancer (27). Nonetheless, the role of RanBPM in tumorigenesis and progression of lung cancer is still unknown.

In the current study, we determined the levels of RanBPM transcripts in human lung cancer tumors along with paired normal lung tissues and analysed its association with clinical features and patient survival. Moreover, we also examined biological functions of RanBPM in lung cells using a knockdown model.

\section{Materials and Methods}

Cell lines. Human lung adenocarcinoma cell line (A549) and human lung squamous cell carcinoma cell line (SKMES1) were purchased from the American Type Culture Collection (Manassas, VA, USA). The cells were routinely cultured in Dulbecco's modified Eagle's medium/F12 (DMEM/F12) supplemented with $10 \%$ foetal calf serum (FCS), amphotericin B, penicillin, streptomycin(Sigma-Aldrich Inc., Poole, Dorset, UK), at $37^{\circ} \mathrm{C}$ with $5 \% \mathrm{CO}_{2}$ and $95 \%$ humidity.

Lung tissues. Lung cancer tissues $(\mathrm{n}=56)$ along with matched normal lung tissues $(\mathrm{n}=56)$ were obtained immediately after surgical resection at the Beijing Cancer Hospital with informed consent from the patients. Tissue samples were stored at the Tissue Bank of Peking University Oncology School. Clinical and histopathological information was recorded. All protocols were reviewed and approved by the Beijing Cancer Hospital Research Ethics Committee (MTA10062009).

$R N A$ isolation and reverse transcription-polymerase chain reaction $(R T-P C R)$. Total RNA was isolated from lung tissues and cells using TRI ${ }^{\circledR}$ Reagent (Sigma-Aldrich Inc., Poole, Dorset, UK). RNA was converted to cDNA using iScript ${ }^{\mathrm{TM}}$ cDNA Synthesis Kit (Bio-Rad Laboratories Ltd., Hercules, CA, USA). Following reverse transcription, PCR was performed using REDTaq ${ }^{\circledR}$ ReadyMix ${ }^{\mathrm{TM}}$ PCR Reaction Mix (Sigma-Aldrich Inc., Poole, Dorset, UK). Primers used in the study; GAPDH sense: 5'-ATGATATCGCCGCGCTCGTC-3', antisense: 5'-CGCTCGGTGAGGATCTTCA-3'; RanBPM sense: 5'ATCGGCCTCTCTCAGAACAA, antisense: 5'-AATTTGGCGGT AGGTCAGTG. Cycling conditions were preheating at $94^{\circ} \mathrm{C}$ for $5 \mathrm{~min}$, followed by $30 \mathrm{cycles}$ of $30 \mathrm{sec}$ at $94^{\circ} \mathrm{C}$ for denaturation, 30 sec at $55^{\circ} \mathrm{C}$ for annealing and $40 \mathrm{sec}$ at $72^{\circ} \mathrm{C}$ for elongation, followed by a final $10 \mathrm{~min}$ extension at $72^{\circ} \mathrm{C}$. The PCR products were separated on a $1 \%$ agarose gel stained with Sybr Safe DNA gel stain (Invitrogen Ltd., Paisley, Scotland, UK). The product was then visualised under ultraviolet light.
Quantitative PCR (QPCR). Real-time quantitative PCR was used to quantify the level of RanBPM transcripts. The reaction was performed on Step One Plus Real-Time PCR system (Applied Biosystems, Waltham, MA, USA), cDNA samples were then examined for RanBPM transcript expression, along with a set of standards and negative controls. Real-time QPCR conditions were $95^{\circ} \mathrm{C}$ for $10 \mathrm{~min}$, followed by 100 cycles of $95^{\circ} \mathrm{C}$ for $10 \mathrm{sec}, 55^{\circ} \mathrm{C}$ for $35 \mathrm{sec}$, and $72^{\circ} \mathrm{C}$ for $10 \mathrm{sec}$. Pairs of primers were designed using Beacon Design software (PREMIER Biosoft, Palo Alto, CA). RanBPM QPCR primers: ZF: 5-TGTGACAAAAAGGGCACAAA, ZR: 5-ACTGAA CCTGACCGTACAAACAGCCAATGACATCACCA. The underlined sequence in the reverse primers was the additional $\mathrm{Z}$ sequence, which is complementary to the universal $\mathrm{Z}$ probe (TCSBiologicals Ltd., Oxford, UK). GAPDH was used as an internal control to normalize RanBPM gene expression.

Construction of ribozyme transgenes and knockdown of RanBPM in Lung cancer cells. Hammerhead ribozymes targeted against human RanBPM were designed based on the secondary structure of the mRNA generated using Zuker's RNA Mfold program. The ribozymes were synthesised and cloned into pEF6/V5-His-TOPO plasmid vector (Invitrogen Ltd., Paisley, Scotland, UK). The ribozymes were then transfected into A549 and SKMES1cells using a Gene PulserXcell ${ }^{\mathrm{TM}}$ electroporation system (Bio-Rad Laboratories Ltd., Hercules, CA, USA). This was followed by selection of successfully transfected cells using selection medium containing $5 \mu \mathrm{g} / \mathrm{ml}$ blasticidin (Sigma-Aldrich Inc., Poole, Dorset, UK) for up to 2 weeks. Following verification, stable transfectants were cultured in maintenance medium containing $0.5 \mu \mathrm{g} / \mathrm{ml}$ blasticidin. The ribozymes that specifically target RanBPM were generated using touchdown PCR with the appropriate primers (Sense: 5'-CTGCAGGTTCGTCCA CGGCCGGGTAGACTGATGA GTCCGTGAGGA, Antisense: 5'-ACTAGTTGCAGCGGCGGCTG AAGCGTTTCGTCCTCACGGACT).

In vitro cell growth assay. Cells were plated into 96-well plates at density of 3,000 cells/well. The cells were then fixed in $4 \%$ formaldehyde after $1,2,3$ and 4 days respectively. $0.5 \%$ crystal violet $(\mathrm{w} / \mathrm{v})$ was used to stain cells. Following washing, the stained crystal violet was dissolved with $10 \%$ (v/v) acetic acid and the absorbance was determined at a wavelength of $540 \mathrm{~nm}$ using a spectrophotometer (Bio-Tek, ELx800, Bio-Tek Instruments, Inc. Winooski, VT, USA). The absorbance of each cell line at day 2, day 3 and day 4 were then normalised against day 1 absorbance.

Cell matrix adhesion assay. This assay was performed following a previously reported method (14). A 96-well plate was pre-coated with $5 \mu \mathrm{g}$ of Matrigel $^{\mathrm{TM}}$ (BD Biosciences, Oxford, UK) and air dried. Following rehydration by serum free medium, 20,000 cells were added to each well. After $45 \mathrm{~min}$ of incubation, non-adherent cells were washed off using PBS buffer. The adhered cells were fixed and stained. Following washing, the stained crystal violet was dissolved with $10 \%(\mathrm{v} / \mathrm{v})$ acetic acid and the absorbance was determined at a wavelength of $540 \mathrm{~nm}$ using the spectrophotometer (Bio-Tek, ELx800).

Wound/migration assay. The cells were seeded at a density of $1,000,000$ per well into a 6-well plate and allowed to reach confluence. The monolayer of cells was then scraped with a fine gauge needle to create a wound. The movement of cells to close the wound was recorded using a time-lapsed video system. Images 
were captured from the computer at the equivalent of $6 \mathrm{~h}$ intervals in real-time and stored as a series of images. The movement of single cells within a colony was analysed by tracking each cells boundary, for each frame in a series, using ImageJ software (https://imagej.nih.gov)

In vitro invasion assay. Transwell inserts (upper chamber) with 8 $\mu \mathrm{m}$ pore size were coated with $50 \mu \mathrm{g}$ of Matrigel (Collaborative Research Products, Bedford, Massachusetts, USA) and air dried. Following rehydration, cells were seeded at a density of 20,000 per insert and allowed to invade for 2 days. After incubation, cells that had migrated through the matrix and adhered to the other side of the inserts were fixed in $4 \%$ formalin, stained with $0.5 \%$ (weight/volume) crystal violet, and dissolved with $10 \%$ (v/v) acetic acid and the absorbance was determined at a wavelength of 540nm using the spectrophotometer (Bio-Tek, ELx800).

Kaplan-Meier survival analysis. An online database was utilized to assess the correlation of RanBPM expression to survival of patients with lung cancer. The database was created with gene expression data and survival information of 1926 patients with lung cancer from the Gene Expression Omnibus (GEO) database. Briefly, RanBPM was entered into the online Kaplan-Meier survival analysis (http://kmplot.com/analysis/index.php?p=service\&start=1) to obtain survival relevance. Hazard ratio (HR), and log-rank $p$ were determined and displayed.

Statistical analysis. Statistical analysis was performed using SPSS software (version 12.5; SPSS, Inc., Chicago, IL, USA). The relationship between RanBPM expression and tumor grade, TNM staging and nodal status was assessed using the Mann-Whitney test for nonparametric data. The statistical comparisons for cellular functional tests were made using Student's two sample $t$-test. Values of $p<0.05$ were considered statistically significant

\section{Results}

RanBPM is up-regulated in lung cancer tissues. The expression of RanBPM was significantly up-regulated in lung cancer tissues $(n=226)$ compared to normal lung tissues $(n=20)$ in an analysis of gene expression array data (GSE31210) (Figure 1) (28). We further evaluated the RanBPM expression in a cohort of 56 lung cancer tissues with paired normal lung tissues using real-time quantitative PCR. Higher mRNA expression levels of RanBPM were also observed in cancer tissues compared to the paired normal lung tissues using Mann-Whitney test and paired $t$-test, respectively ( $p=0.0011$ and $p=0.0011$ ). According to the tumor staging, T3 tumors exhibited a higher RanBPM expression compared with its expression inT1 tumors $(p=0.0081)$. The relationships between RanBPM expression and histology, clinical TNM staging, lymph node status and differentiation status were also analysed, Statistical analysis showed no significant difference among different groups (Table I).

The prognostic value of RanBPM in Lung cancer. We examined the prognostic value of RanBPM mRNA

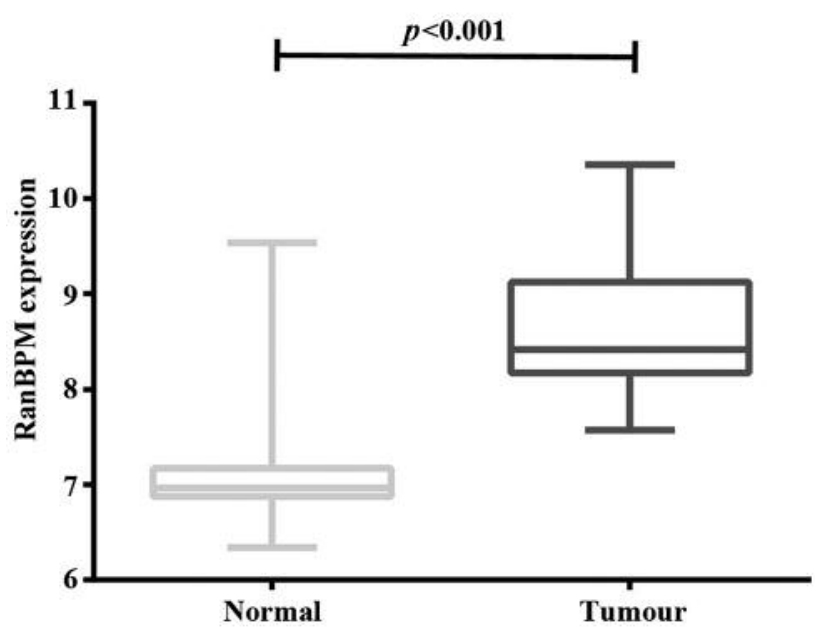

Figure 1. Expression of RanBPM in normal and lung cancer tissue. RanBPM expression is significantly up-regulated in lung cancer compared with its expression in normal lung tissues based on an analysis of a GEO dataset (GSE31210,202582_s_at) (28).

expression using the KMplot (https://www.kmplot.com) (29). The Affymetrix ID for RanBPM is 202582_s_at. RanBPM mRNA low expression was discovered to be linked to worse overall survival (OS) in all lung cancer patients. Median survival of patients with lower RanBPM expression was 57 months compared to 112.7 months of patients with higher expression of the gene. RanBPM expression is also associated with disease free survival of patients with lung cancer (Figure 2A). Median of first progression survival (FP) was 12 months for patients with lower RanBPM expression, $p<0.001$ compared to 26.3 months of patients with higher expression (Figure 2B). Moreover, the reduced expression of RanBPM is significantly associated with poorer OS of patients with lung adenocarcinomas (Figure 2C), but not squamous cell carcinomas (Figure 2D).

Creation of lung cancer cell sub-lines with RanBPM knockdown. To further dissect the role of RanBPM in lung cancer, we performed a knockdown of RanBPM using antiRanBPM ribozyme in a lung adenocarcinoma cell line (A549) and a lung squamous cell carcinoma cell line (SKMES1). RanBPM-knockdown was successfully established in both SKMES1 (SKMES1 RanRib) and A549 (A549 RanRib) cell lines after transfection with antiRanBPM ribozyme transgenes and compared to empty vectors (PEF) as controls. Reduced expression of RanBPM in the cell lines was verified using RT-PCR (Figure 3). The RanBPM-knockdown cells were used for the following in vitro studies. 
A

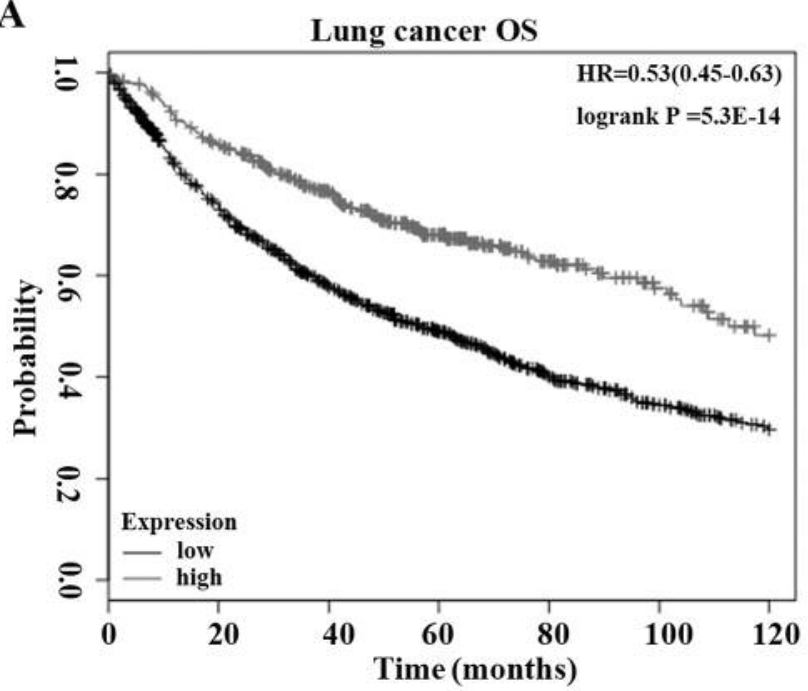

C

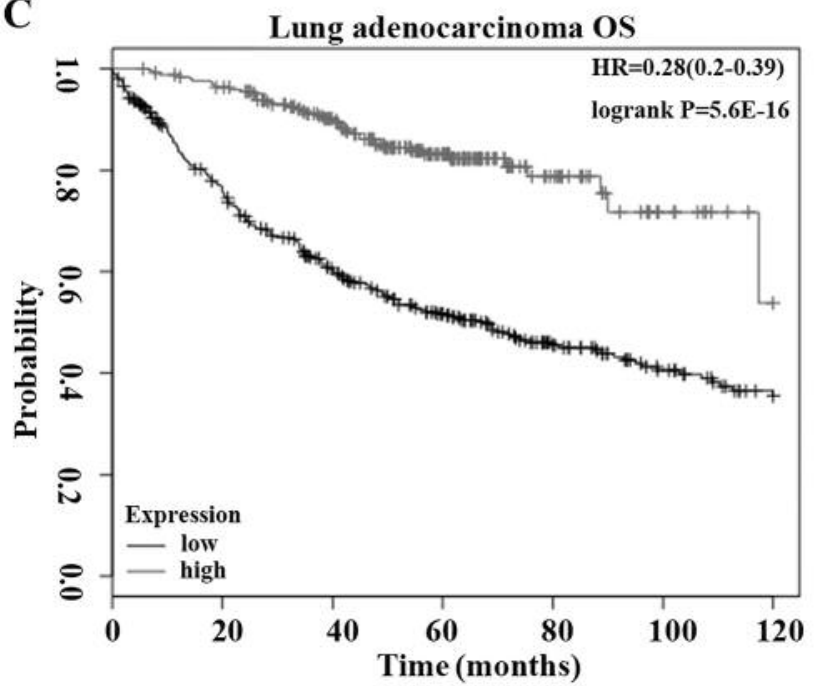

B

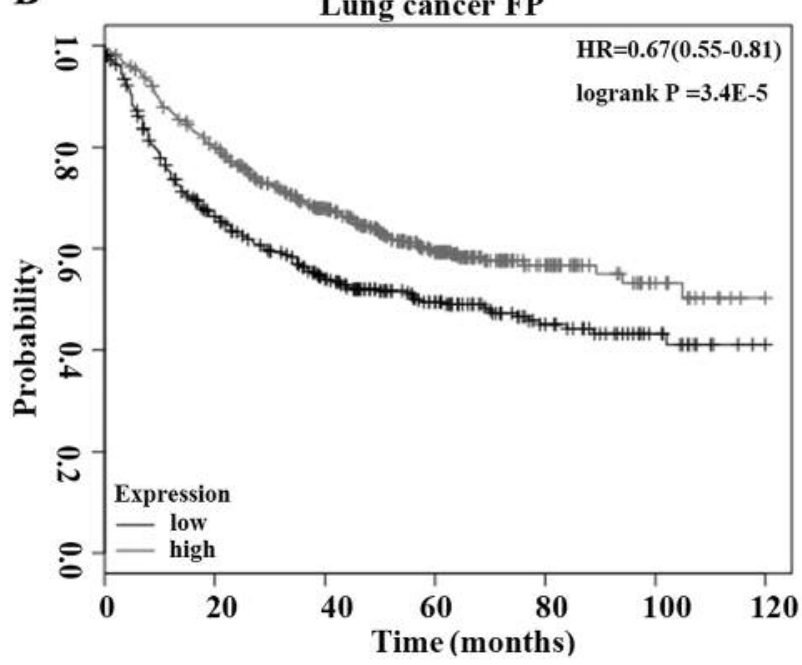

D

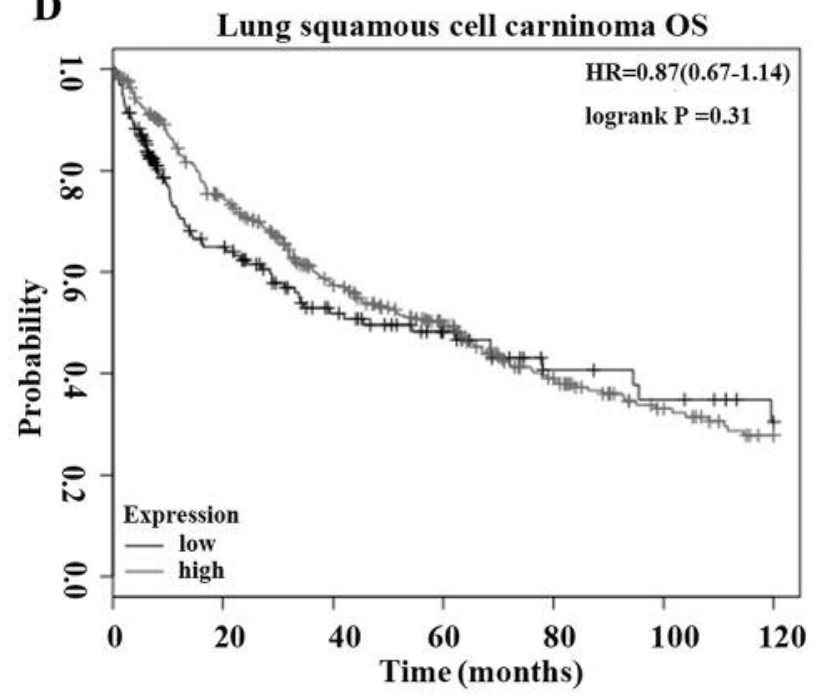

Figure 2. Expression of RanBPM and overall survival (OS) or first progression (FP) of patients with lung cancer. A: Association of RanBPM with OS of LC patients. B: Association of RanBPM with FP of LC patients. C: Association of RanBPM with OS of patients with lung adenocarcinoma. D: Association of RanBPM with OS of patients with squamous cell lung cancer.

Influence of RanBPM-knockdown on cell proliferation and adhesion. We first determined the effect of RanBPM knockdownon in vitro cell growth of these two lung cancer cell lines. An increase was seen in the growth of A549 RanBPMknockdown cells, $(p<0.001)$ compared to the control (Figure 4A). A similar change was also observed in SKMES1RanRib cells, $(p<0.001)$ compared to the control (Figure 4B).

We further examined the influence of RanBPM on the adhesive nature of these lung cancer cells. RanBPM knockdown in SKMES1 significantly reduced the adhesive properties $(p<0.05)$ compared to the control cells (Figure 4C).
In contrast, knockdown of RanBPM expression resulted in a slight increase of adhesive ability in A549 cells (Figure 4D).

RanBPM is involved in the invasion and migration of lung cancer cells. We tested the influence of RanBPM on the invasiveness of both A549 and SKMES1 cell lines. A significant increase was seen in the invasion of A549 RanBPM-knockdown cells compared with its control (Figure 5A). However, little effect was seen on the invasion of SKMES1 following the knockdown of RanBPM, though a subtle increase was also noticed but to a much less extent 
Table I. RanBPM expression and clinicopathological characteristics of lung cancer.

\begin{tabular}{|c|c|c|c|c|}
\hline Clinical/pathological features & Sample no. & Median (Copy no.) & IQR & $p$-Value \\
\hline Tumour tissue & 56 & 119 & $15-860$ & \\
\hline Background tissue & 56 & 13.7 & $3.2-74.8$ & 0.0011 \\
\hline Paired tumour tissue & 56 & 119 & $15-860$ & \\
\hline Paired background tissue & 56 & 13.7 & $3.2-74.8$ & 0.0011 \\
\hline \multicolumn{5}{|l|}{ Histology } \\
\hline Squamous carcinoma & 18 & 92 & $3-570$ & \\
\hline Adenocarcinoma & 26 & 286 & $18-1201$ & 0.1736 \\
\hline Others & 12 & N/A & N/A & \\
\hline \multicolumn{5}{|l|}{ TNM staging } \\
\hline I & 14 & 90 & $10-365$ & \\
\hline II & 12 & 484 & $24-1323$ & 0.2578 \\
\hline III & 16 & 199 & $31-495$ & 0.3496 \\
\hline \multicolumn{5}{|l|}{ Tumour differentiation } \\
\hline HighMed & 11 & 186 & $0-981$ & \\
\hline Med & 13 & 206 & $25-1066$ & 0.39 \\
\hline MedLow & 8 & 260 & $45-1899$ & 0.61 \\
\hline \multicolumn{5}{|l|}{ Tumour status } \\
\hline $\mathrm{T} 1$ & 11 & 44.3 & $5.7-146$ & \\
\hline $\mathrm{T} 2$ & 13 & 206 & $37-923$ & 0.1178 \\
\hline $\mathrm{T} 3$ & 15 & 512 & $186-1458$ & 0.0081 \\
\hline \multicolumn{5}{|l|}{ Lymph node status } \\
\hline NO & 32 & 96 & $6-882$ & \\
\hline N1 & 7 & 444 & $47-865$ & 0.46 \\
\hline $\mathrm{N} 2$ & 12 & 199 & $45-1412$ & 0.83 \\
\hline
\end{tabular}

(Figure 5B). Interestingly, the knockdown of RanBPM elicited contrasting effects on the migration of these two cell lines. A remarkably increased migration was seen in RanBPM knockdown SKMES1 cells while a decrease was observed in the A549 RanBPM knockdown cells in comparison with the respective control (Figure 5C and D).

\section{Discussion}

RanBPM is ubiquitously expressed in human and murine cell lines and has been observed in the cytoplasm, nucleus and plasma membrane. Overexpression of RanBPM has been evident in certain cancers and is also associated with the invasion of certain cancers $(20,21)$. In the present study, we investigated the role of RanBPM in lung cancer. In the analysis of a publically available gene expression array dataset (GSE31210), an increased expression of RanBPM was shown in lung cancers compared to normal lung tissues. Our quantitative analysis of RanBPM transcripts in a cohort of lung cancers showed an elevated expression of RanBPM in the lung cancers in comparison with paired normal lung tissue controls. However, further analyses of its association with patients' survival using an online Kaplan-Meier survival analysis of a combined cohort of lung cancer patients showed that those with low RanBPM expression tumors had shorter

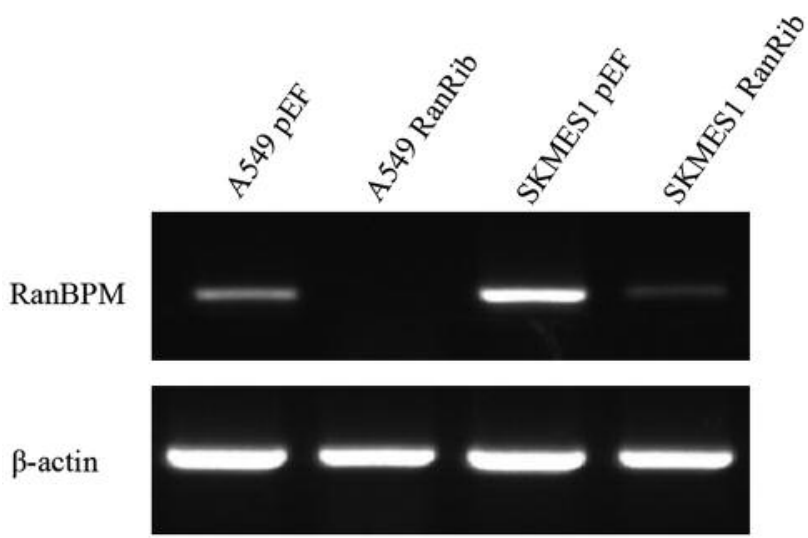

Figure 3. Knockdown of RanBPM in lung cancer cell lines. Knockdown of RanBPM in A549 and SKMES1 cells was verified using reverse transcription-quantitative polymerase chain reaction. A549pEF and SKMES1pEF are A549 and SKMES1 cells transfected with empty plasmids, respectively. A549RanRib and SKMES1RanRib are A549 and SKMES1 cells transfected with anti-RanBPM ribozyme transgenes, respectively.

OS and FP compared to patients with high RanBPM expression. More interestingly, the association with survival is highly significant in patients with adenocarcinoma, but not the other common type of non-small cell lung cancer, i.e. 
A

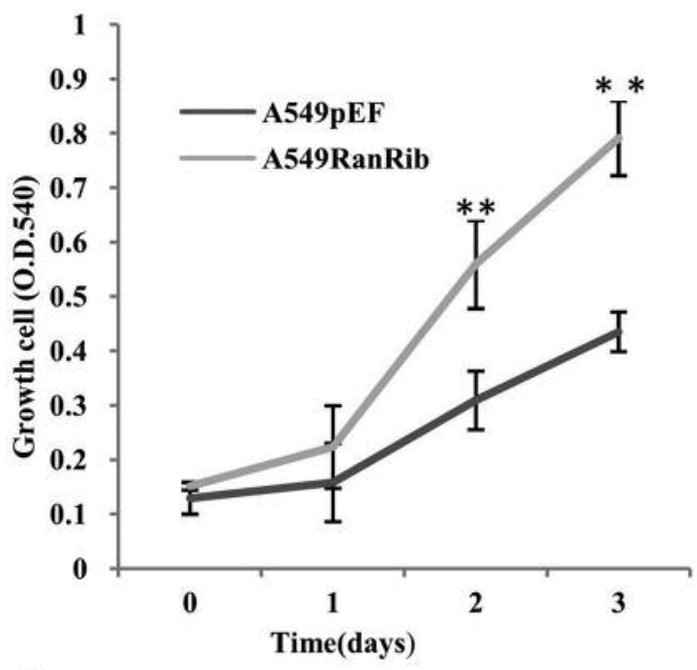

C

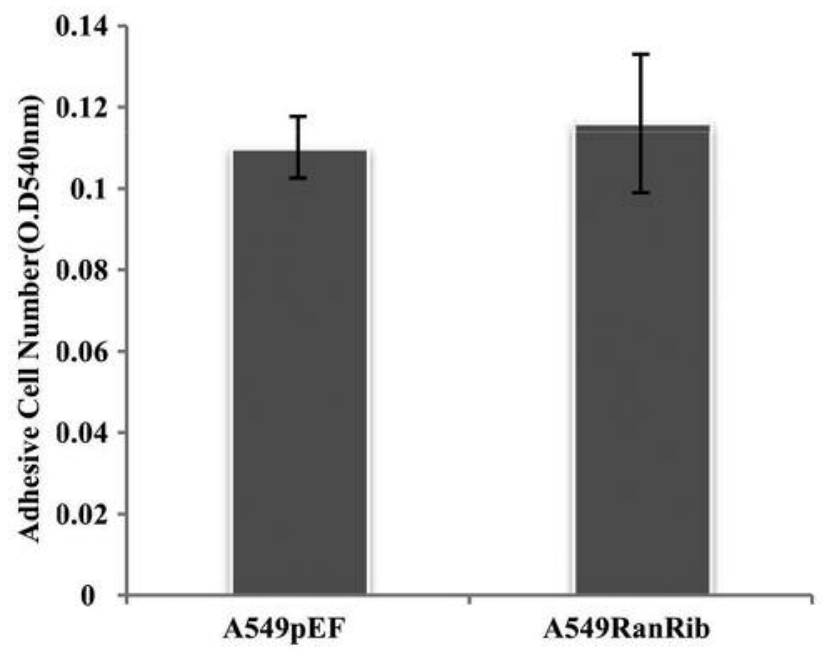

B

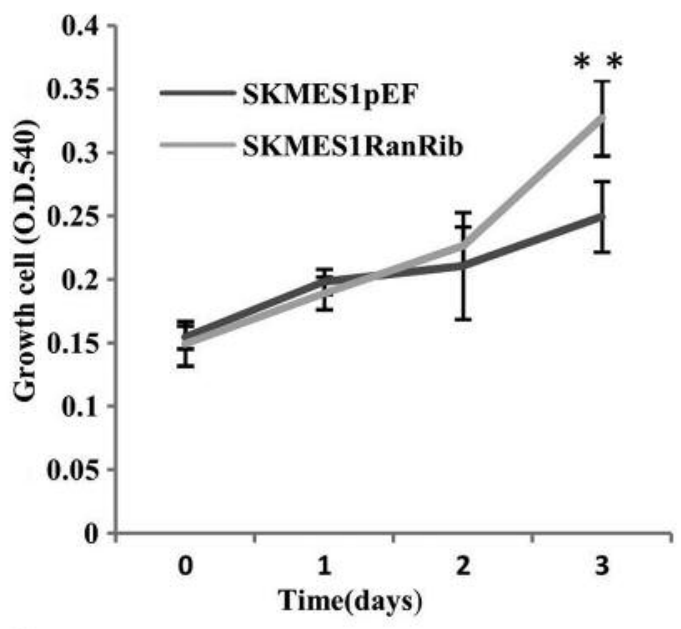

D

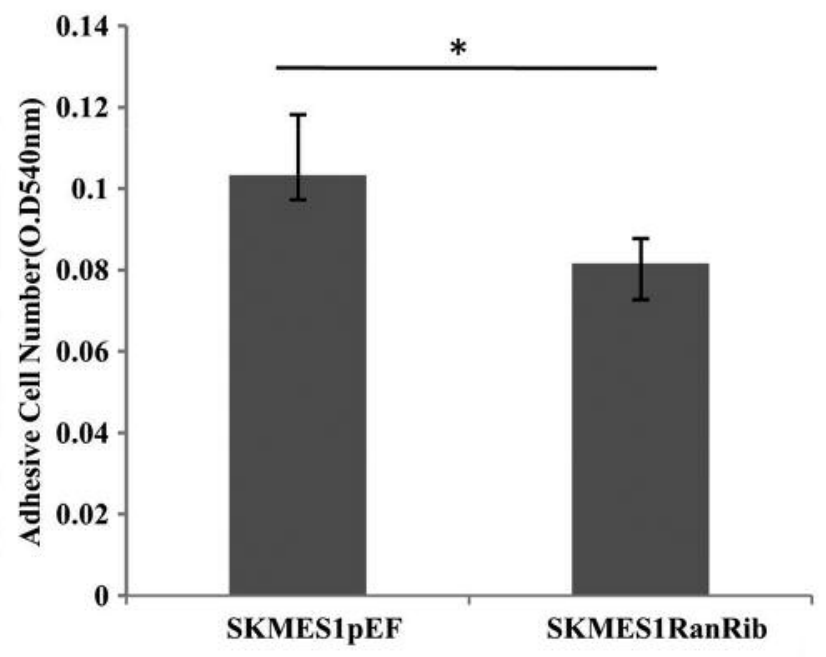

Figure 4. Influence on in vitro growth and adhesion of A549 and SKMES1 cells by RanBPM knockdown. A: Growth of A549RanRib cells was increased compared to A549pEF control ( $p<0.001$ at day3). B: Growth of SKMES1RanRib cells was increased compared to SKMES1pEF control $(p<0.001$ at day 3). C: Cell adhesion of A549RanRib cells was slightly increased. D: SKMES1RanRib cells had a remarkable decrease in cell adhesion $(p<0.05)$. *represents $p<0.05$ and $* *$ represents $p<0.01$.

squamous cell lung cancer. It suggests that RanBPM plays an inhibitory role, though it is up-regulated in lung cancers. This result might also provide us with a new view to explain the difference in prognosis between lung adenocarcinoma and lung squamous cell carcinoma patients.

RanBPM has been implicated in the control of a multitude of cellular processes including regulation of cell motility (25), adhesion, development $(30,31)$, transcription (32-35), and apoptosis (36-38). For a better understanding of the role played by RanBPM in lung cancer, we employed two lung cancer cell lines, an adenocarcinoma cell line (A549) and a squamous cell carcinoma cell line (SKMES1) to examine its involvement in the regulation of certain cellular functions. Both cell lines express RanBPM. We then established two RanBPM knockdown cell lines to further evaluate the consequent effect on cellular functions. The adhesion assays demonstrated that the knockdown of RanBPM resulted in decreased lung squamous carcinoma cell adhesion, but exhibited little effect on the lung adenocarcinoma cell line A549. This means that the role of RanBPM in different types of lung cancer may be different. Our previous study of RanBPM in gastric cancer cell lines showed that the 
A

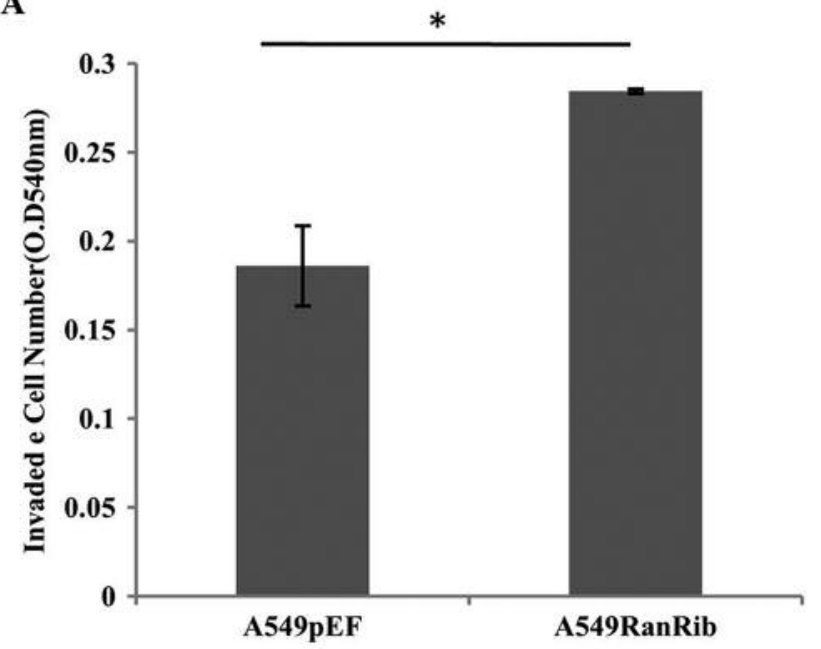

$\mathbf{C}$

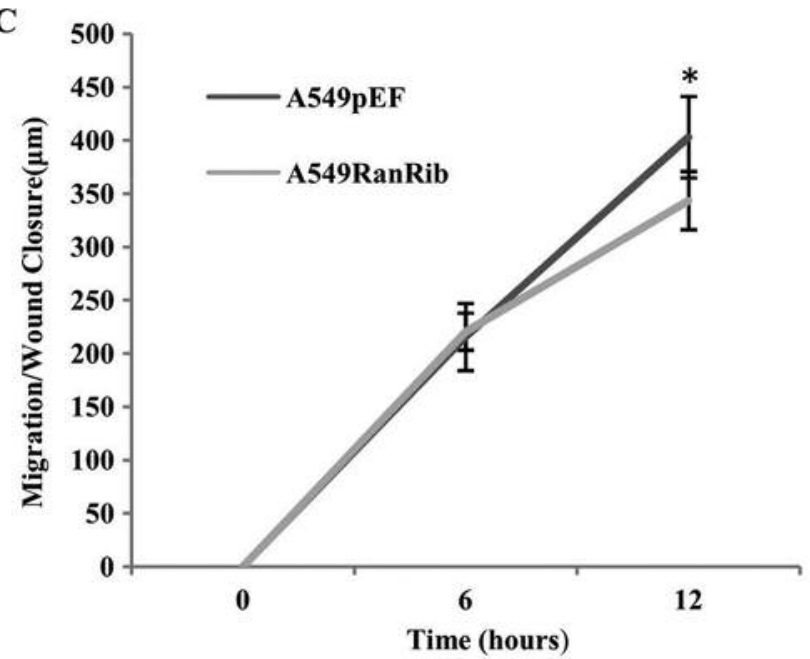

B

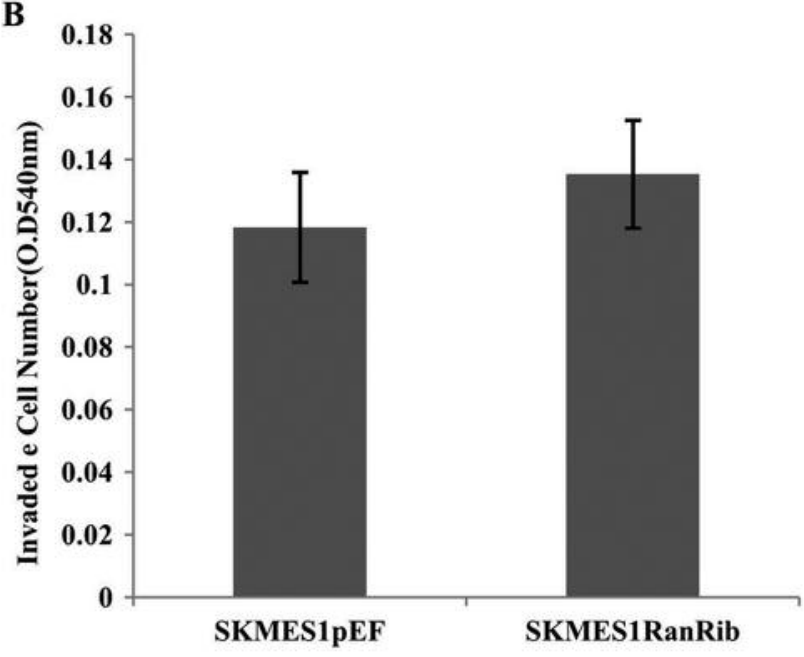

D

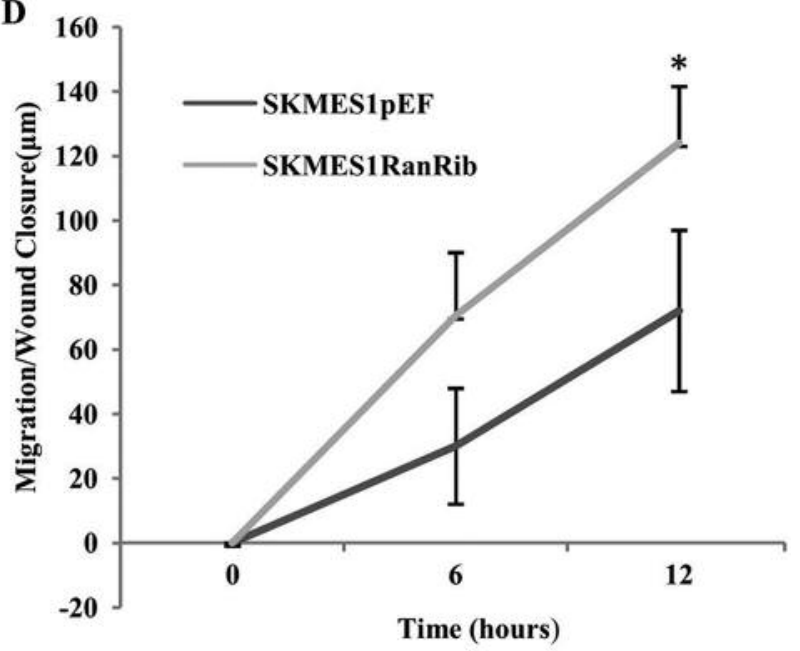

Figure 5. Effect of RanRib knockdown on invasion and migration of A549 and SKMES1 cells. A: Invasion was enhanced in A549RanRib cells, $p=0.02$ compared to control cells. B: SKMES1RanRibcells also showed a slightly increased invasion, $p=0.6$ compared to control. C: Decreased migration was seen in A549RanRib cells compared to A549pEF control $(p<0.05)$. D: SKMES1RabRib cells migrated further in comparison with SKMES1pEF cells over a period of $12 \mathrm{~h} .(p<0.05)$, *represents $p<0.05$.

knockdown of RanBPM resulted in a reduction of adhesion (20). These findings collectively suggest that RanBPM is involved in the regulation of cell adhesion through an unknown mechanism.

More interestingly, differential effects were also observed in the two lung cancer cell lines when we assessed the migration using a wound assay. The loss of RanBPM in the lung adenocarcinoma cancer cells (A549) resulted in a slightly decreased migration, whereas a marked increase was seen in the RanBPM-knockdown SKMES1 cells. A recent study showed that RanBPM inhibits BLT2-mediated IL-8 production and invasiveness in aggressive breast cancer cells
(21). In our experiments, the knockdown of RanBPM promoted the invasion of A549 cells which was not observed in the SKMES1 cells. Moreover, we found that knockdown of RanBPM can increase the proliferation of both A549 and SKMES1 cells. The differential effects on the cellular functions are in line with findings for its association with patients' survival. Its inhibitory role for proliferation, migration and invasion of lung cancer cells, in particular the adenocarcinoma cells, provides supportive evidence for its clinical relevance, i.e. the association between low RanBPM expression and disease progression and also poor prognosis of the disease. 
A gene expression array analysis of RanBPM knockdown cells suggested that RanBPM played an inhibitory role in cancer cells by targeting various pathways including ERK, Wnt, Notch and PI3K/Akt pathways (19). Following knockdown of RanBPM, up-regulations of RON tyrosine kinase, L1CAM and ELF3 were seen in both Hela and HCT116 cell lines. The involvement of these pathways and molecules in the RanBPM-regulated cellular functions in lung cancer cells requires further investigation.

Recently, Palmieri et al. reported that RanBP9 is a novel mediator of the cellular DDR, whose accumulation into the nucleus upon IR is dependent on ATM kinase activity (27). As known, several cancer treatments, and lung cancer in particular, cause DNA damage and activation of the cellular response to DNA damage. In our previous study, we found that RanBPM plays a role in differential responses to certain chemotherapeutic agents and consequently influences tumor chemoresistance (20). An implication has been suggested for this molecule in the treatment of lung cancer using radiotherapy and chemotherapy. Reduced expression of RanBPM confers a support to the cancer cells under these treatments. Therefore, it can be speculated that RanBPMrelated downstream pathway/molecules can serve as a target to eliminate resistance. Our next step will be to study the specific molecular mechanisms of RanBPM in different lung cancers as well as its upstream and downstream regulatory factors. In addition to these, S100A7, also known as psoriasin can interact with RanBPM (39). Overexpression of Psoriasin has also been observed in lung cancers which is associated with poor prognosis of the disease (40). Interaction between S100A7 and RanBPM in lung cancer warrants a further investigation.

Taken together, our results showed that RanBPM is significantly involved in the lung cancer development and disease progression, acting as a tumor suppressor. Reduced expression of RanBPM is associated with disease progression and poor prognosis. RanBPM plays an inhibitory role in the regulation of proliferation and invasion of lung cancer cells, in particular the adenocarcinoma cells. Further investigations may provide concept proof for novel targeted lung cancer therapies.

\section{Acknowledgements}

The Authors would like to thank Cancer Research Wales and Albert Hung Foundation for their kind support. Mr Zehang Zhao is a recipient of the Chinese Medical Research Studentship of Cardiff University.

\section{References}

1 Siegel RL, Miller KD and Jemal A: Cancer statistics, 2015. CA Cancer J Clin 65(1): 5-29, 2015.

2 Chen W, Zheng R, Baade PD, Zhang S, Zeng H, Bray F, Jemal $\mathrm{A}, \mathrm{Yu} \mathrm{XQ}$ and He J: Cancer statistics in china, 2015. CA Cancer J Clin 66(2): 115-132, 2016.
3 Cooper WA, Lam DC, O'Toole SA and Minna JD: Molecular biology of lung cancer. J Thorac Dis 5(Suppl 5): S479-490, 2013.

4 Hiret S, Senellart $\mathrm{H}$ and Bennouna J: Molecular biology of lung cancer series. Rev Mal Respir 27(8): 954-958, 2010.

5 Akita H: Molecular biology of lung cancer. Nihon Kokyuki Gakkai Zasshi 42(5): 378-386, 2004.

6 Pendharkar D, Ausekar BV and Gupta S: Molecular biology of lung cancer-a review. Indian J Surg Oncol 4(2): 120-124, 2013.

7 Larsen JE and Minna JD: Molecular biology of lung cancer: Clinical implications. Clin Chest Med 32(4): 703-740, 2011.

8 Nana-Sinkam SP and Powell CA: Molecular biology of lung cancer: Diagnosis and management of lung cancer, 3rd ed: American college of chest physicians evidence-based clinical practice guidelines. Chest 143(5 Suppl): e30S-39S, 2013.

9 Suda K and Mitsudomi T: [molecular biology for surgical treatment of lung cancer]. Kyobu Geka 70(1): 4-8, 2017.

10 Dai Y and Han B: [research advance on molecular biology of lung cancer metastasis.]. Zhongguo Fei Ai Za Zhi 11(5): 747751, 2008.

11 Hanibuchi M, Kim SJ, Fidler IJ and Nishioka Y: The molecular biology of lung cancer brain metastasis: An overview of current comprehensions and future perspectives. J Med Invest 61(3-4): 241-253, 2014.

12 Zarogoulidis P, Baka S, Labaki S, Lazaridis G and Trakada G: Targeted lung cancer treatments and eye metastasis. Med Hypothesis Discov Innov Ophthalmol 6(1): 10-13, 2017.

13 Nakamura M, Masuda H, Horii J, Kuma K, Yokoyama N, Ohba $\mathrm{T}$, Nishitani H, Miyata T, Tanaka $\mathrm{M}$ and Nishimoto $\mathrm{T}$ : When overexpressed, a novel centrosomal protein, ranbpm, causes ectopic microtubule nucleation similar to gamma-tubulin. J Cell Biol 143(4): 1041-1052, 1998.

14 Nishitani H, Hirose E, Uchimura Y, Nakamura M, Umeda M, Nishii $\mathrm{K}$, Mori $\mathrm{N}$ and Nishimoto T: Full-sized ranbpm cdna encodes a protein possessing a long stretch of proline and glutamine within the n-terminal region, comprising a large protein complex. Gene 272(1-2): 25-33, 2001.

15 Stambolic V, Suzuki A, de la Pompa JL, Brothers GM, Mirtsos C, Sasaki T, Ruland J, Penninger JM, Siderovski DP and Mak TW: Negative regulation of pkb/akt-dependent cell survival by the tumor suppressor pten. Cell 95(1): 29-39, 1998.

16 Shin SY, Rath O, Choo SM, Fee F, McFerran B, Kolch W and Cho KH: Positive- and negative-feedback regulations coordinate the dynamic behavior of the ras-raf-mek-erk signal transduction pathway. J Cell Sci 122(Pt 3): 425-435, 2009.

17 Murrin LC and Talbot JN: Ranbpm, a scaffolding protein in the immune and nervous systems. J Neuroimmune Pharmacol 2(3): 290-295, 2007.

18 Atabakhsh E and Schild-Poulter C: Ranbpm is an inhibitor of erk signaling. PLoS One 7(10): e47803, 2012.

19 Atabakhsh E, Wang JH, Wang X, Carter DE and Schild-Poulter C: Ranbpm expression regulates transcriptional pathways involved in development and tumorigenesis. Am J Cancer Res 2(5): 549-565, 2012.

20 Shao S, Sun PH, Satherley LK, Gao X, Ji KE, Feng YI, Jia Y, Ji J, Jiang WG and Ye L: Reduced ranbpm expression is associated with distant metastasis in gastric cancer and chemoresistance. Anticancer Res 36(3): 1295-1303, 2016.

21 Wei JD, Jang JH and Kim JH: Ranbpm inhibits blt2-mediated il8 production and invasiveness in aggressive breast cancer cells. Biochem Biophys Res Commun 483(1): 305-311, 2017. 
22 Woo JA, Roh SE, Lakshmana MK and Kang DE: Pivotal role of ranbp9 in integrin-dependent focal adhesion signaling and assembly. FASEB J 26(4): 1672-1681, 2012.

23 Umeda M, Nishitani $\mathrm{H}$ and Nishimoto T: A novel nuclear protein, twa1, and muskelin comprise a complex with ranbpm. Gene 303: 47-54, 2003.

24 Chang Y, Paramasivam M, Girgenti MJ, Walikonis RS, Bianchi $\mathrm{E}$ and LoTurco JJ: Ranbpm regulates the progression of neuronal precursors through m-phase at the surface of the neocortical ventricular zone. Dev Neurobiol 70(1): 1-15, 2010.

25 Wang D, Li Z, Messing EM and Wu G: Activation of ras/erk pathway by a novel met-interacting protein ranbpm. J Biol Chem 277(39): 36216-36222, 2002.

26 Guimaraes-Gomes V, Oliveira-Carvalho AL, Junqueira-deAzevedo IL, DL SD, Pujol-Luz M, Castro HC, Ho PL and Zingali RB: Cloning, characterization, and structural analysis of a c-type lectin from bothrops insularis (bil) venom. Arch Biochem Biophys 432(1): 1-11, 2004.

27 Palmieri D, Scarpa M, Tessari A, Uka R, Amari F, Lee C, Richmond T, Foray C, Sheetz T, Braddom A, Burd CE, Parvin JD, Ludwig T, Croce CM and Coppola V: Ran binding protein 9 (ranbp9) is a novel mediator of cellular DNA damage response in lung cancer cells. Oncotarget 7(14): 18371-18383, 2016.

28 Okayama H, Kohno T, Ishii Y, Shimada Y, Shiraishi K, Iwakawa R, Furuta K, Tsuta K, Shibata T, Yamamoto S, Watanabe S, Sakamoto H, Kumamoto K, Takenoshita S, Gotoh N, Mizuno H, Sarai A, Kawano S, Yamaguchi R, Miyano S and Yokota J: Identification of genes upregulated in alk-positive and egfr/kras/alk-negative lung adenocarcinomas. Cancer Res 72(1): 100-111, 2012.

29 Gyorffy B, Surowiak P, Budczies J and Lanczky A: Online survival analysis software to assess the prognostic value of biomarkers using transcriptomic data in non-small-cell lung cancer. PLoS One 8(12): e82241, 2013.

30 Scantlebury N, Zhao XL, Rodriguez Moncalvo VG, Camiletti A, Zahanova S, Dineen A, Xin JH and Campos AR: The drosophila gene ranbpm functions in the mushroom body to regulate larval behavior. PLoS One 5(5): e10652, 2010.

31 Dansereau DA and Lasko P: Ranbpm regulates cell shape, arrangement, and capacity of the female germline stem cell niche in drosophila melanogaster. J Cell Biol 182(5): 963-977, 2008.
32 Brunkhorst A, Karlen M, Shi J, Mikolajczyk M, Nelson MA, Metsis $\mathrm{M}$ and Hermanson O: A specific role for the tfiid subunit taf4 and ranbpm in neural progenitor differentiation. Mol Cell Neurosci 29(2): 250-258, 2005.

33 Chang LK, Liu ST, Kuo CW, Wang WH, Chuang JY, Bianchi E and Hong YR: Enhancement of transactivation activity of rta of epstein-barr virus by ranbpm. J Mol Biol 379(2): 231-242, 2008.

34 Rao MA, Cheng H, Quayle AN, Nishitani H, Nelson CC and Rennie PS: Ranbpm, a nuclear protein that interacts with and regulates transcriptional activity of androgen receptor and glucocorticoid receptor. J Biol Chem 277(50): 48020-48027, 2002.

35 Poirier MB, Laflamme L and Langlois MF: Identification and characterization of ranbpm, a novel coactivator of thyroid hormone receptors. J Mol Endocrinol 36(2): 313-325, 2006.

36 Kramer S, Ozaki T, Miyazaki K, Kato C, Hanamoto T and Nakagawara A: Protein stability and function of p73 are modulated by a physical interaction with ranbpm in mammalian cultured cells. Oncogene 24(5): 938-944, 2005.

37 Atabakhsh E, Bryce DM, Lefebvre KJ and Schild-Poulter C: Ranbpm has proapoptotic activities that regulate cell death pathways in response to DNA damage. Mol Cancer Res 7(12): 1962-1972, 2009.

38 Mikolajczyk M, Shi J, Vaillancourt RR, Sachs NA and Nelson M: The cyclin-dependent kinase 11(p46) isoform interacts with ranbpm. Biochem Biophys Res Commun 310(1): 14-18, 2003.

39 Emberley ED, Gietz RD, Campbell JD, HayGlass KT, Murphy LC and Watson PH: Ranbpm interacts with psoriasin in vitro and their expression correlates with specific clinical features in vivo in breast cancer. BMC Cancer 2: 28, 2002.

$40 \mathrm{Hu} \mathrm{M}$, Ye L, Ruge F, Zhi X, Zhang L and Jiang WG: The clinical significance of psoriasin for non-small cell lung cancer patients and its biological impact on lung cancer cell functions. BMC Cancer 12: 588, 2012.

Received May 22, 2017

Revised June 1, 2017

Accepted June 2, 2017 